\title{
Does Telomere Shortening Precede the Onset of Hypertension in Spontaneously Hypertensive Mice?
}

\author{
Christine L. Chiu, Nerissa L. Hearn, Devin Paine, Nicole Steiner, and Joanne M. Lind \\ Western Sydney University, School of Medicine, Sydney, New South Wales, Australia
}

\begin{abstract}
Telomere length is widely considered as a marker of biological aging. Clinical studies have reported associations between reduced telomere length and hypertension. The aim of this study was to compare telomere length in hypertensive and normotensive mice at pre-disease and established disease time points to determine whether telomere length differs between the strains before and after the onset of disease. Genomic DNA was extracted from kidney and heart tissues of 4-, 12-, and 20-week-old male hypertensive (BPH/2J) and normotensive (BPN/3J) mice. Relative telomere length (T/S) was measured using quantitative PCR. Age was inversely correlated with telomere length in both strains. In 4-week-old pre-hypertensive animals, no difference in T/S was observed between BPH/2J and BPN/3J animals in kidney or heart tissue (kidney $p=0.14$, heart $p=0.06$ ). Once the animals had established disease, at 12 and 20 weeks, BPH/2J mice had significantly shorter telomeres when compared to their age-matched controls in both kidney (12 weeks $p<0.001$ and 20 weeks $p=0.004$ ) and heart tissues (12 weeks $p<0.001$ and 20 weeks $p<0.001$ ). This is the first study to show that differences in telomere lengths between $\mathrm{BPH} / 2 \mathrm{~J}$ and BPN/3J mice occur after the development of hypertension and do not cause hypertension in the $\mathrm{BPH} / 2 \mathrm{~J}$ mice.
\end{abstract}

Keywords: Schlager mice, hypertension, telomere length

Telomeres are repetitive DNA sequences that are located at both ends of each chromosome. They function to protect the ends of the chromosome, maintaining genome stability and integrity. In somatic cells, telomeres shorten with every cell division, and when telomeres reach a critical length, they activate DNA damage checkpoints, which in turn induce senescence or apoptosis. Telomere shortening occurs during human aging and as such telomere length is widely considered as a marker of biological ageing (Blackburn, 2001). Telomere length is influenced by a number of endogenous factors such as cell division, oxidative stress, and inflammation, as well as by environmental factors including poor lifestyle (Valdes et al., 2005) and psychological stress (O’Donovan et al., 2012).

Clinical studies have reported associations between shorter leukocyte telomere length and cardiovascular diseases such as hypertension (Demissie et al., 2006; Yang et al., 2009) and diabetes (Astrup et al., 2010). These studies measured telomere length in individuals with established disease and in leukocytes where rapid cell division frequently occurs. Although studies have demonstrated that the rate of telomere attrition is similar across different tissues within an individual (Daniali et al., 2013; Kuznetsova et al., 2010), they do not tell us whether the individuals who end up with disease had shorter telomeres prior to the onset of disease. A study in telomerase-deficient mice $\left(\operatorname{Terc}^{-/-}\right)$has shown a link between shorter telomeres and hypertension (Perez-Rivero et al., 2006). These mice show progressive telomere shortening in successive generations. First generation $\mathrm{Terc}^{-/}$mice have long telomeres and normal phenotypes, whereas third generation $\mathrm{Terc}^{-/-}$have significantly shorter telomeres and increased blood pressure (Perez-Rivero et al., 2006). Taken together, this would suggest that telomere shortening could contribute to the development of hypertension.

The BPH/2J mice are a genetic model of hypertension developed in the 1970s by Schlager (1974). These mice were selectively bred for elevated blood pressure alongside a normotensive (BPN/3J) line, from a base population of eight inbred strains of mice. The BPN/3J strain is a widely accepted control for the hypertensive $\mathrm{BPH} / 2 \mathrm{~J}$ strain (Davern et al., 2009; Friese et al., 2005; Jackson et al., 2014). The BPH/2J strain parallels human hypertension, with increased blood pressure, heart rate, endothelial dysfunction, and reduced survival, compared to the normotensive

RECEIVED 18 May 2016; ACCEPTED 15 June 2016. First published online 12 August 2016.

AdDRESS FOR CORRESPONDENCE: J. Lind, School of Medicine, Western Sydney University, Locked bag 1797, Penrith, Sydney, NSW 2751, Australia. E-mail: j.lind@westernsydney.edu.au 
BPN/3J control strain (Schlager \& Sides, 1997). The BPH/2J mice are pre-hypertensive at 4 weeks (Friese et al., 2005) and have been shown to exhibit increased blood pressure at 7 weeks of age, when compared to the normotensive controls (BPN/3J) (Schlager \& Sides, 1997). We have previously shown that at 12 weeks of age, systolic blood pressure is on average $34 \mathrm{mmHg}$ higher in $\mathrm{BPH} / 2 \mathrm{~J}$ male mice, compared with BPN/3J mice of the same age (Chiu et al., 2014). Radiotelemetry studies on 20-week-old males have also shown that blood pressure in $\mathrm{BPH} / 2 \mathrm{~J}$ mice is consistently high and in BPN/3J mice is consistently normal (Davern et al., 2009; Jackson et al., 2014). The genetic backgrounds of the two strains are not identical and there is evidence that variation in candidate genes, such as Hmgcr (Sonawane et al., 2011) and Agtr1a (Wong et al., 2003), contribute to the differences in phenotype between the two strains. Apart from these single gene studies, the underlying genetic basis of disease in the $\mathrm{BPH} / 2 \mathrm{~J}$ strain is not known.

We hypothesize that spontaneously hypertensive Schlager mice will have shorter telomeres compared to age-matched normotensive controls. This shortening will precede the onset of hypertension and may contribute to the high blood pressure seen in these animals. The aim of this study was to measure and compare telomere length in kidney and heart tissues in hypertensive and normotensive mice at pre-disease and established disease time points to determine whether telomere length differs between the strains before and after the onset of disease.

\section{Methods}

\section{Animal Model and Tissue Collection}

This study involved Schlager BPH/2J and BPN/3J mice. We and others have previously used radiotelemetry to show that the BPH/2J strain consistently exhibits high blood pressure at 12 and 20 weeks of age (Chiu et al., 2014; Davern et al., 2009; Jackson et al., 2014; Schlager \& Sides, 1997). To avoid introducing changes in telomere length and gene expression due to stress handling from blood pressure measurement, blood pressure was not directly measured in the animals used in this study. Kidney and heart tissues were collected from male $\mathrm{BPH} / 2 \mathrm{~J}$ and $\mathrm{BPN} / 3 \mathrm{~J}$ mice during the inactive period, which corresponds to the trough in $\mathrm{di}$ urnal blood pressure, at 4,12 , and 20 weeks of age $(n=$ $8 /$ age/strain). Mice were euthanized via cervical dislocation and tissues were snap frozen in liquid nitrogen and stored at $-80^{\circ} \mathrm{C}$. This study had the University of Western Sydney Animal Ethics Committee approval (ARA7980) and all efforts were made to minimize suffering.

\section{Relative Telomere Length (T/S)}

Tissues were mechanically disrupted in the deep frozen state and genomic DNA was isolated using the Qiagen Blood and Tissue Mini Kit (Qiagen, Germany) according to the manufacturer's recommendations, quantified using the NanoPhotometer (Implen, Germany) and stored at $-80^{\circ} \mathrm{C}$. Telomere repeat copy number (T) to single-copy gene copy number $(\mathrm{S})$ ratio (T/S ratio) was determined by real-time quantitative PCR using the Power SYBR Mix (Applied Biosystems, Life Technologies USA), as previously described (Callicott \& Womack, 2006). It uses the acidic ribosomal phosphoprotein PO (36B4) gene as the single copy gene, as it is well conserved and has been used for gene dosage studies (Callicott \& Womack, 2006). The PCR reactions were carried out in a MxPro3005P Real Time PCR System (Stratagene, Agilent Technologies, USA) under the following conditions $95^{\circ} \mathrm{C}-10 \mathrm{~min} ; 40$ cycles $\left(95^{\circ} \mathrm{C}-30 \mathrm{sec}\right.$; $60^{\circ} \mathrm{C}-1 \mathrm{~min}$ ); followed by a dissociation curve. All samples were run in triplicate. Cycle threshold $(\mathrm{Ct})$ values for each sample were calculated using the MxPRo QPCR software (Stratagene, Agilent Technologies, USA). Triplicate Ct values were averaged and the quantity of each sample was calculated using the delta-delta Ct method. Telomere length was given as the T/S ratio. To minimize plate-to-plate variation, comparisons between groups were limited to samples that were run on the same plate.

\section{Quantitative PCR}

RNA was extracted from homogenized tissue, as previously described. cDNA was synthesized using the SensiFAST cDNA synthesis kit (Bioline, AUS) as per the manufacturer's recommendations. Telomerase reverse transcriptase (Tert), telomerase RNA component (Terc), and beta actin (bAct) expression were quantified using the following Taqman assays; mTert Mm00436931_m1, mTerc Mm01261365_s1, bAct Mm00607939_s1, and the Taqman ${ }^{\circledR}$ Gene Expression Master mix (Applied Biosystems, Life Technologies, USA) as per manufacturer's protocol. All samples were run in triplicate, and triplicate $\mathrm{Ct}$ values were averaged and the quantity of each sample was calculated using the delta-delta $\mathrm{Ct}$ method.

\section{Statistical Analysis}

Statistical analysis was performed with PASW Statistics version 22 and GraphPad Prism version 6. Analyses of variance using Tukey's multiple comparisons test was used to compare telomere length between ages within the same strain and an adjusted $p$ value of $<0.05$ was considered significant. Analysis of covariance was used to test whether there was a difference in the rate of telomere shortening between the strains. A general linear model was used to compare data between hypertensive $(\mathrm{BPH} / 2 \mathrm{~J})$ and normotensive (BPN/3J) groups at each time point using a significance level of $p<0.01$ to account for multiple comparisons. Results are expressed as mean \pm standard error.

\section{Results}

This study measured relative telomere length in kidney and heart tissues in male $\mathrm{BPH} / 2 \mathrm{~J}$ and $\mathrm{BPN} / 3 \mathrm{~J}$ mice at a 
A

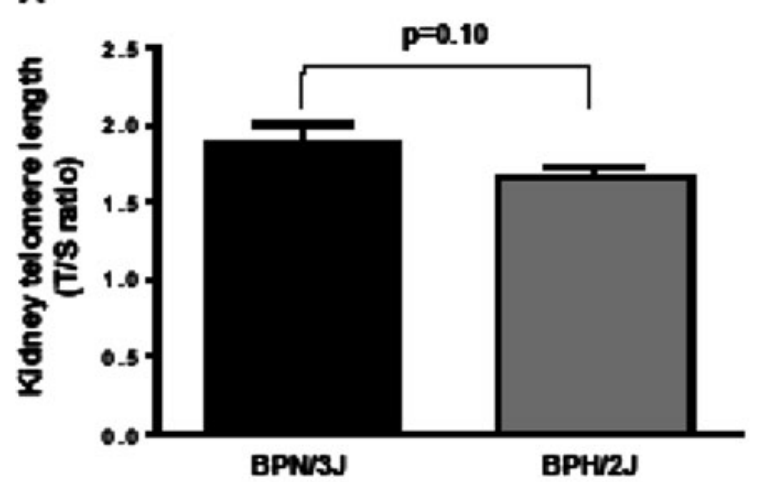

C

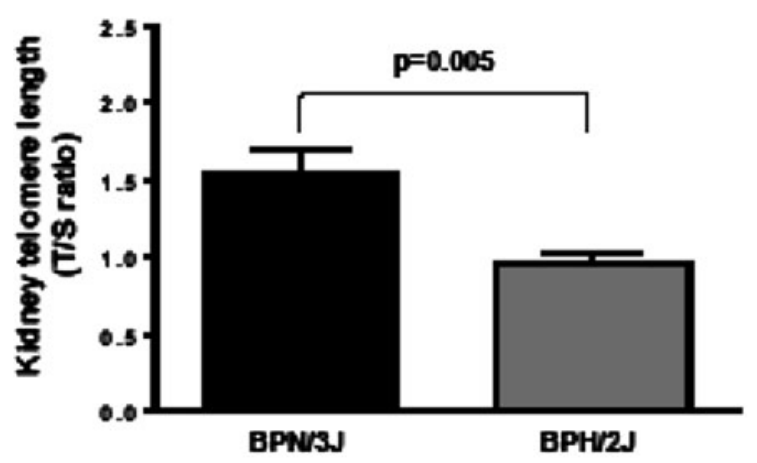

E

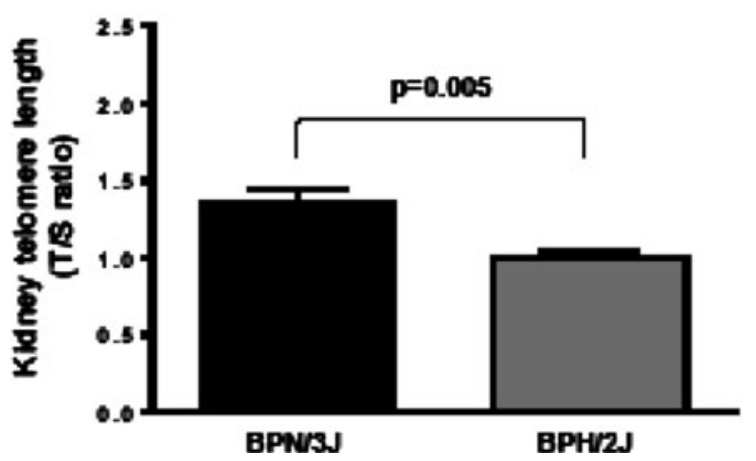

B

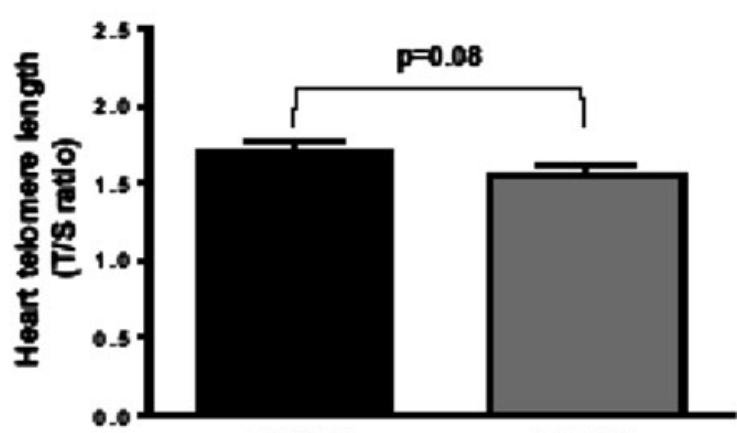

BPNYY

BPHr2J

D

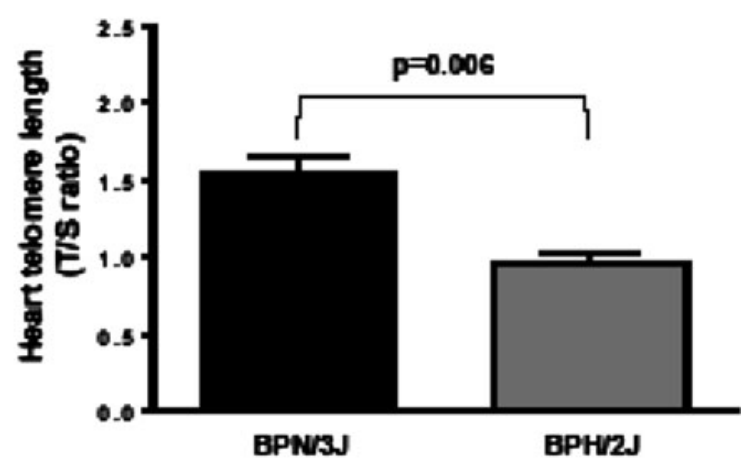

$\mathbf{F}$

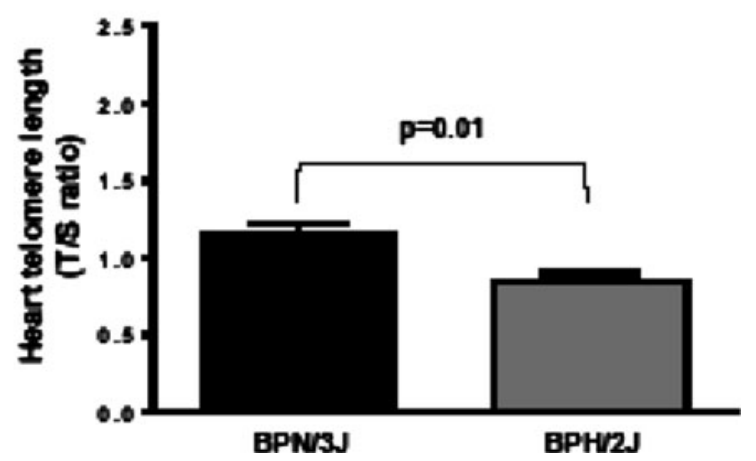

\section{FIGURE 1}

Telomere length (T/S) ratio in kidney and heart tissues from BPNH/3J and BPH/2J mice at 4, 12, and 20 weeks of age ( $n=8 / \mathrm{strain} /$ age). Notes: (A) Kidney, 4 weeks. (B) Kidney, 12 weeks. (C) Kidney, 20 weeks. (D) Heart, 4 weeks. (E) Heart, 12 weeks. (F) Heart, 20 weeks. Data expressed as mean $\pm \mathrm{SE}$.

pre-hypertensive age and during established disease. In 4week-old pre-hypertensive animals, no significant difference in telomere length was observed between $\mathrm{BPH} / 2 \mathrm{~J}$ and $\mathrm{BPN} / 3 \mathrm{~J}$ kidney or heart tissue (Figure $1 \mathrm{~A}$ and B). At 12 and 20 weeks of age, established disease time points, significant differences in telomere length were observed between $\mathrm{BPH} / 2 \mathrm{~J}$ and $\mathrm{BPN} / 3 \mathrm{~J}$ mice (Figure $1 \mathrm{C}-\mathrm{F}$ ). BPH/2J mice had significantly shorter telomeres when compared to age matched BPN/3J normotensive controls. This pattern was consistent in kidney and heart tissues. When we compared telomere length within strains, 4-week BPN/3J kidneys had significantly longer telomeres when compared to 20 -week
BPN/3J kidneys (Figure 2A). In BPN/3J hearts, 4-week hearts had significantly longer telomeres when compared to 12- and 20-week hearts (Figure 2B). In BPH/2J kidneys, telomeres were significantly longer in 4-week kidneys when compared to 12- and 20-week kidneys (Figure 2C). Similarly, in BPH/2J hearts, telomeres were significantly longer in 4-week hearts when compared to 12- and 20-week hearts (Figure 2D). There was also a statistically significant difference in the rate of telomere length shortening between hypertensive and normotensive groups in both kidney $(p<$ $0.001)$ and heart $(p<0.001)$, with a faster rate of shortening in the hypertensive group. 

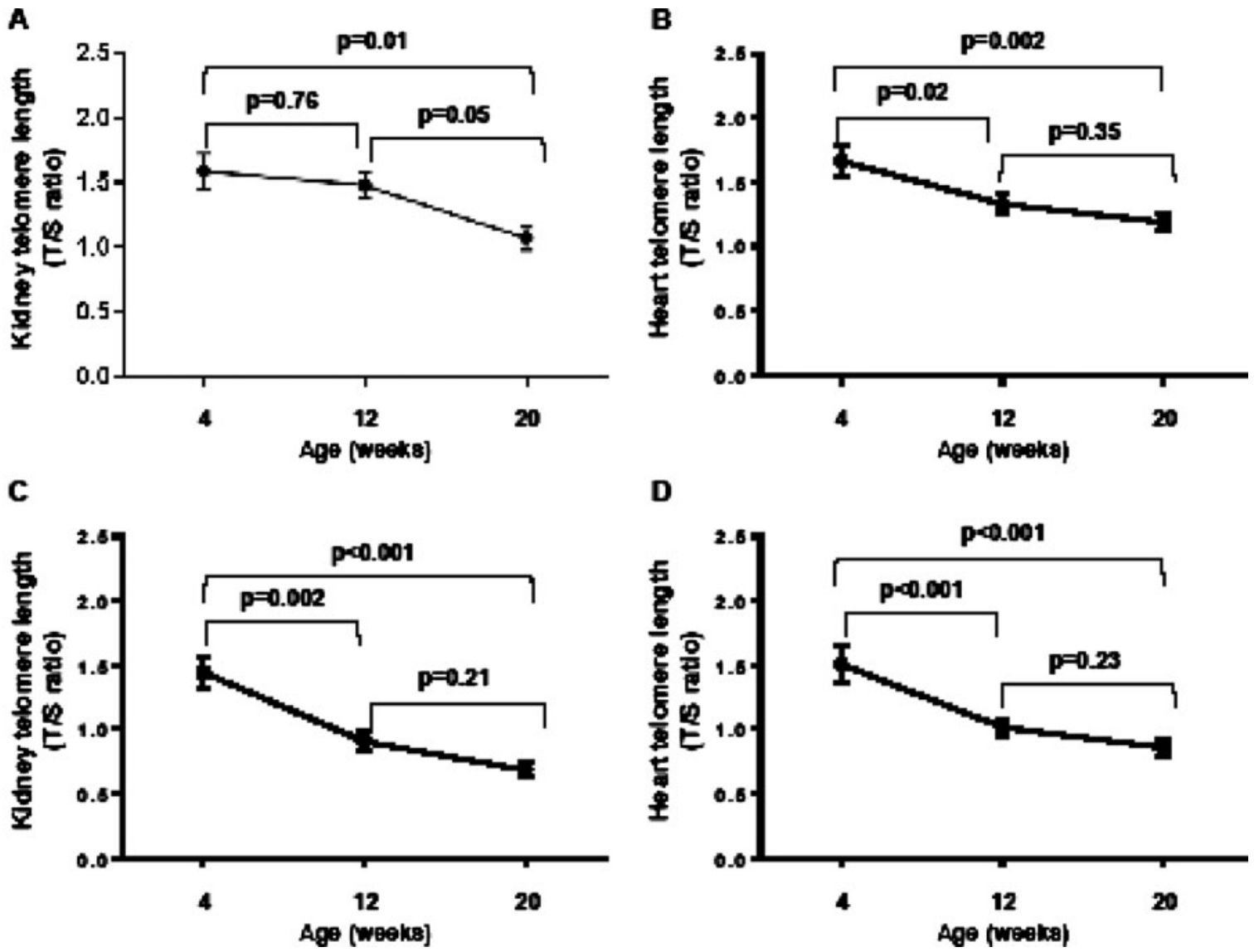

\section{FIGURE 2}

Telomere length (T/S ratio) in kidney and heart tissues between age groups over time in BPN/3J and BPH/2 mice $(n=8 / \mathrm{strain} / \mathrm{age}$ ). Notes: (A) Kidney telomere length in BPN/3J mice. (B) Heart telomere length in BPN/3J mice. (C) Kidney telomere length in BPH/2J mice. (D) Heart telomere length in $\mathrm{BPH} / 2 \mathrm{~J}$ mice. Data expressed as mean $\pm \mathrm{SE}$ and adjusted $p$ values are reported.

The expression of telomerase subunits Tert and Terc were measured in kidney and heart tissues in BPN/3J and BPH/2J mice. The expression of Tert in the kidney did not differ between strains at any time point (Figure 3A, C, and E). In heart tissue, little ( $\mathrm{Ct} \geq 38)$ or no Tert expression was observed at any time point. Terc expression in the kidney and heart did not differ between strains at any time point (Figure 3B, D, and F).

\section{Discussion}

This study aimed to determine if $\mathrm{BPH} / 2 \mathrm{~J}$ hypertensive mice had shorter telomeres when compared to age-matched BPN/3J controls, and whether shorter telomeres occurred prior to the onset of hypertension in these mice. Telomere length in pre-hypertensive $\mathrm{BPH} / 2 \mathrm{~J}$ mice did not differ when compared to age-matched BPN/3J mice in either tissue. At an established disease time point, telomere length was significantly shorter in $\mathrm{BPH} / 2 \mathrm{~J}$ mice compared to their normotensive controls in both kidney and heart tissues. Within strains, telomere length decreased with age, which is consistent with reports that show telomere length decreases with increased age (Blackburn, 2001).

Numerous clinical studies have associated shorter telomeres with cardiovascular diseases, including hypertension (Demissie et al., 2006; Yang et al., 2009), type 1 and 2 diabetes (Astrup et al., 2010; Zhao et al., 2013), and stroke (Jiang et al., 2013). The majority of these studies have been cross-sectional and were unable to determine whether shorter telomeres are present prior to disease onset. This is the first study to compare telomere length in $\mathrm{BPH} / 2 \mathrm{~J}$ and $\mathrm{BPN} / 3 \mathrm{~J}$ prior to disease and after the onset of disease.

The BPH/2J hypertensive mice are pre-hypertensive at 4 to 5 weeks of age (Friese et al., 2005), with blood pressure increases occurring at 7 weeks of age (Schlager \& Sides, 1997). We and others have previously shown that blood pressure is significantly higher in $\mathrm{BPH} / 2 \mathrm{~J}$ mice at 12 and 20 weeks of age when compared to age-matched BPN/3J mice (Chiu et al., 2014; Jackson et al., 2014). In the present study, we show that there is no significant difference in telomere length in the kidney or heart prior to the onset of hypertension, and differences in 
A

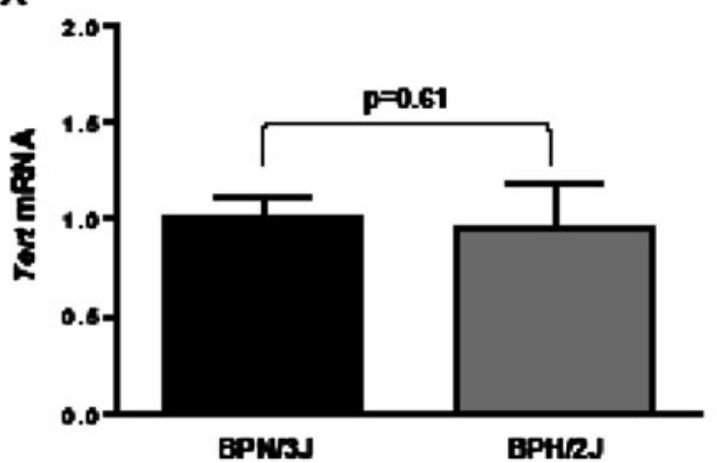

C

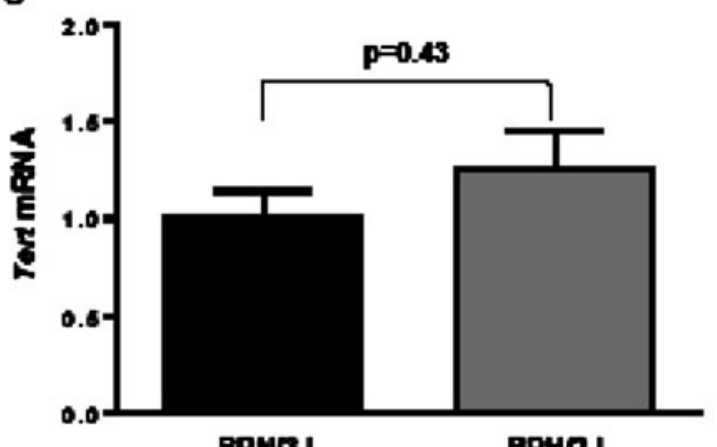

BPWBS

E

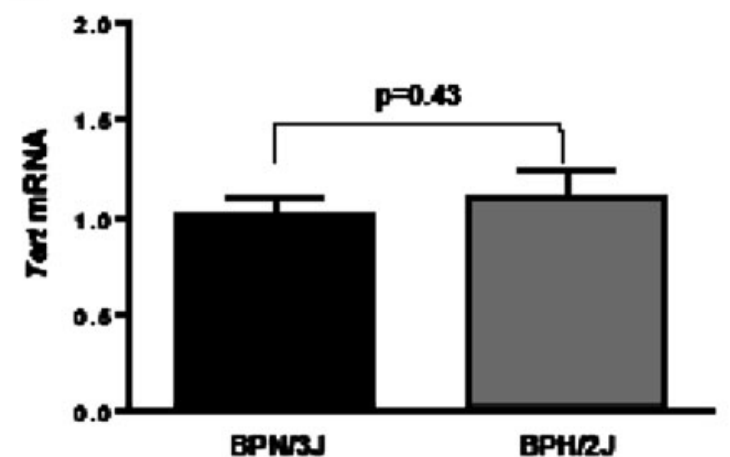

B
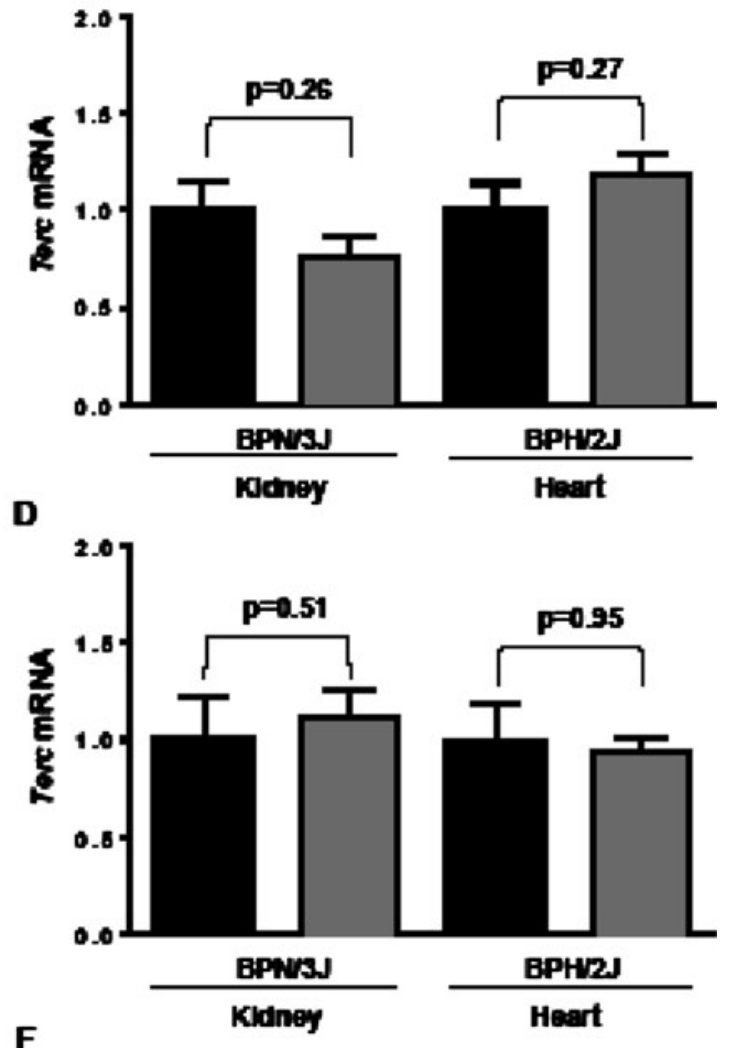

$\mathbf{F}$

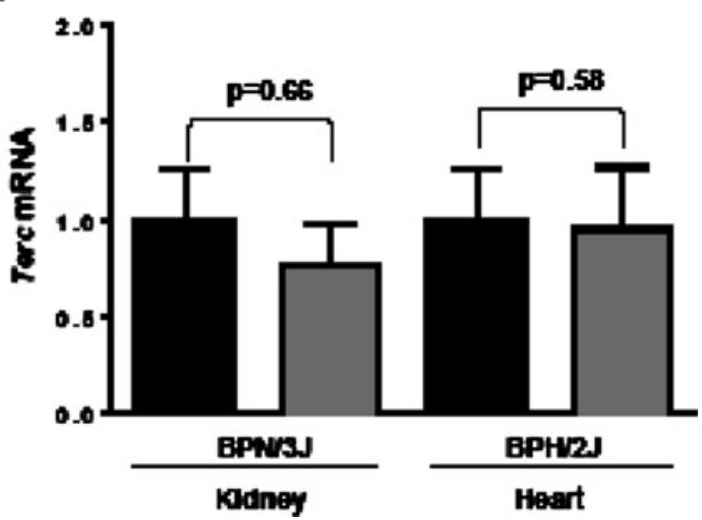

\section{FIGURE 3}

The expression of telomerase genes Tert and Terc in kidney and heart tissues from BPN/3J and BPH/2J mice, with expression plotted relative to BPN/3J. Notes: (A) Expression of Tert mRNA in 4-week-old kidneys relative to BPN/3J. (B) Expression of Tert mRNA in 12-weekold kidneys relative to BPN/3J. (C) Expression of Tert mRNA in 20-week-old kidneys relative to BPN/3J. (D) Expression of Terc mRNA in 4-week kidneys and hearts. (E) Expression of Terc mRNA in 12-week kidneys and hearts. (F) Expression of Terc mRNA in 20-week kidneys and hearts. Data expressed as mean $\pm \mathrm{SE}$.

telomere length are only evident after the onset of disease. This indicates that the shorter telomeres in $\mathrm{BPH} / 2 \mathrm{~J}$ mice are associated with the presence of hypertension in these animals.

Alterations in genes that maintain telomere length during the cell cycle may also contribute to the telomere attrition observed in the $\mathrm{BPH} / 2 \mathrm{~J}$ mice. Telomerase, the enzyme that synthesizes and maintains telomeres, is made up of two components, telomerase reverse transcriptase (Tert) and the telomerase RNA component (Terc). In the present study, no difference in Tert or Terc expression was observed between the two strains, this would suggest that telomerase dysfunction is unlikely to contribute to reduced telomere length in the $\mathrm{BPH} / 2 \mathrm{~J}$ mice.

In this study, telomere length was measured in kidney and heart tissues as these organs are major regulators of blood pressure. Although multiple cell types are present within these tissues, previous studies have shown that 
age-dependent telomere shortening is similar across different tissues within an individual, particularly between proliferative (leukocytes) and low proliferative (muscle) tissues (Daniali et al., 2013; Kuznetsova et al., 2010). However, these studies do not address whether the rate of telomere shortening across organs remains similar during disease. We observed similar patterns of telomere shortening in kidney and heart tissues within the BPH/2J strain, which is consistent with previous findings (Daniali et al., 2013).

Environmental factors that are known to modulate telomere length, including lifestyle and cardiovascular risk factors, make it difficult to determine the relationship between telomere length and disease. These lifestyle risk factors may also contribute to reduced telomere length. However, the use of inbred mouse strains in this study helped to decrease the effect of environmental confounders. A limitation of our study was that telomere length could not be measured within the same animal before and after the onset of hypertension. The use of inbred mouse strains and identical environmental conditions partially overcame this problem. Furthermore, the use of quantitative PCR to measure relative telomere length must also be acknowledged as a limitation; however, it has been reported that this method is reliable and the results are comparable to other techniques (Marques et al., 2015). Studies have also reported that telomere length can vary depending on the method of DNA extraction (Denham et al., 2014). To minimize variation, all samples were collected and extracted using the same method, and samples that were directly compared were run on the same plate. Finally, although there are similarities between mice and humans for association between telomere attrition and aging, rodent telomeres are significantly longer (5 to 10 times longer) than human telomeres and this difference should be taken into account when interpreting the result of these studies in relation to human phenotypes (Calado \& Dumitriu, 2013).

The BPH/2J mice are a model of neurogenic hypertension and have been shown to exhibit exaggerated activity of the sympathetic nervous system in response to adverse stress (Davern et al., 2010). Clinical studies have associated chronic psychological stress and anxiety disorders with accelerated telomere shortening (Hoen et al., 2013; O’Donovan et al., 2012) and increased risk of hypertension (Bacon et al., 2014). Production of reactive oxygen species and oxidative stress has also been shown to mediate increased sympathetic nervous activity, leading to increased blood pressure (Ye et al., 1997). Oxidative stress plays an important role in the pathophysiology of hypertension, with the generation of reactive oxygen species in the kidneys being a contributing factor in the development of disease (Mathis et al., 2012; Taylor et al., 2006). Studies in Dahl saltsensitive rats and spontaneously hypertensive rats (SHR), both established animal models of hypertension, have reported increased levels of oxidative stress with reduced telomere lengths in the kidney (Baumann et al., 2008) and heart (Oyama et al., 2012). Furthermore, treatment with an angiotensin II receptor antagonist was associated with reduced oxidative stress and increased telomere length in the SHR (Baumann et al., 2008). Oxidative stress has also been associated with reduced telomere length, with increases in levels of reactive oxygen species correlating with reduced telomere length (Richter \& von Zglinicki, 2007).

In the BPN/3J and BPH/2J strains, gene expression profiling in 12-week-old animals have reported that fatty acid oxidation processes and oxidoreductase activity pathways are enriched in the heart and kidneys of BPH/2J animals (Chiu et al., 2014; Puig et al., 2010). This would indicate that oxidative stress pathways are activated in the hypertensive $\mathrm{BPH} / 2 \mathrm{~J}$ animals. Therefore, we propose that the pathways that regulate sympathetic vascular tone in response to adverse stress and the production of reactive oxygen species in response to stress could also contribute to shorter telomeres. Investigation of markers of oxidative stress in these animals and whether ablation of the production of reactive oxygen species can prevent telomere shortening are required.

In summary, contrary to our original hypothesis, the $\mathrm{BPH} / 2 \mathrm{~J}$ hypertensive mice develop shorter telomeres after the onset of hypertension. Further studies are needed to determine whether the difference in telomere length is a product of increased oxidative stress caused by stress, age, and disease, or other mechanisms.

\section{Acknowledgments}

The authors would like to thank the Western Sydney University School of Medicine Animal Facility staff for their assistance in animal care and tissue collection. JML is supported by a National Health and Medical Research - Australian Biomedical Fellowship. None of the authors have any conflicts of interest to report.

\section{Authors' Contributions}

CLC participated in study design, laboratory work, data analysis and interpretation, and manuscript writing. NLH, DP and NS participated in laboratory work. JML participated in study design, data interpretation, and manuscript writing. All authors read and approved the final manuscript.

\section{References}

Astrup, A. S., Tarnow, L., Jorsal, A., Lajer, M., Nzietchueng, R., Benetos, A., ... Parving, H. H. (2010). Telomere length predicts all-cause mortality in patients with type 1 diabetes. Diabetologia, 53, 45-48.

Bacon, S. L., Campbell, T. S., Arsenault, A., \& Lavoie, K. L. (2014). The impact of mood and anxiety disorders on incident hypertension at one year. International Journal of $\mathrm{Hy}$ pertension, 2014, Article ID 953094. 
Baumann, M., Bartholome, R., Peutz-Kootstra, C. J., Smits, J. F., \& Struijker-Boudier, H. A. (2008). Sustained tubulointerstitial protection in SHRs by transient losartan treatment: An effect of decelerated aging? American Journal of Hypertension, 21, 177-182.

Blackburn, E. H. (2001). Switching and signaling at the telomere. Cell, 106, 661-673.

Calado, R. T., \& Dumitriu, B. (2013). Telomere dynamics in mice and humans. Seminars in Hematology, 50, 165-174.

Callicott, R. J., \& Womack, J. E. (2006). Real-time PCR assay for measurement of mouse telomeres. Comparative Medicine, 56, 17-22.

Chiu, C. L., Jackson, K. L., Hearn, N. L., Steiner, N., Head, G. A., \& Lind, J. M. (2014). Identification of genes with altered expression in male and female Schlager hypertensive mice. BMC Medical Genetics, 15, 101.

Daniali, L., Benetos, A., Susser, E., Kark, J. D., Labat, C., Kimura, M., ... Aviv, A. (2013). Telomeres shorten at equivalent rates in somatic tissues of adults. Nature Communications, 4, 1597.

Davern, P. J., Jackson, K. L., Nguyen-Huu, T. P., La Greca, L., \& Head, G. A. (2010). Cardiovascular responses to aversive and nonaversive stressors in Schlager genetically hypertensive mice. American Journal of Hypertension, 23, 838-844.

Davern, P. J., Nguyen-Huu, T. P., La Greca, L., Abdelkader, A., \& Head, G. A. (2009). Role of the sympathetic nervous system in Schlager genetically hypertensive mice. Hypertension, 54, 852-859.

Demissie, S., Levy, D., Benjamin, E. J., Cupples, L. A., Gardner, J. P., Herbert, A., ... Aviv, A. (2006). Insulin resistance, oxidative stress, hypertension, and leukocyte telomere length in men from the Framingham heart study. Aging Cell, 5, 325-330.

Denham, J., Marques, F. Z., \& Charchar, F. J. (2014). Leukocyte telomere length variation due to DNA extraction method. BMC Research Notes, 7, 877.

Friese, R. S., Mahboubi, P., Mahapatra, N. R., Mahata, S. K., Schork, N. J., Schmid-Schonbein, G. W., \& O'Connor, D. T. (2005). Common genetic mechanisms of blood pressure elevation in two independent rodent models of human essential hypertension. American Journal of Hypertension, 18, 633-652.

Hoen, P. W., Rosmalen, J. G., Schoevers, R. A., Huzen, J., van der Harst, P., \& de Jonge, P. (2013). Association between anxiety but not depressive disorders and leukocyte telomere length after 2 years of follow-up in a population-based sample. Psychological Medicine, 43, 689697.

Jackson, K. L., Nguyen-Huu, T. P., Davern, P. J., \& Head, G. A. (2014). Energy metabolism in BPH/2J genetically hypertensive mice. Hypertension Research, 37, 413-421.

Jiang, X., Dong, M., Cheng, J., Huang, S., He, Y., Ma, K., ... Guo, Y. (2013). Decreased leukocyte telomere length (LTL) is associated with stroke but unlikely to be causative. PLoS One, 8, e68254.

Kuznetsova, T., Codd, V., Brouilette, S., Thijs, L., Gonzalez, A., Jin, Y., ... Samani, N. J. (2010). Association between left ventricular mass and telomere length in a population study. American Journal of Epidemiology, 172, 440-450.

Marques, F. Z., Booth, S. A., Prestes, P. R., Curl, C. L., Dellbridge, L. M., Lewandowski, P., ... Charchar, F. J. (2015). Telomere dynamics during aging in polygenic left ventricular hypertrophy. Physiological Genomics, 48, 42-49.

Mathis, K. W., Venegas-Pont, M., Masterson, C. W., Stewart, N. J., Wasson, K. L., \& Ryan, M. J. (2012). Oxidative stress promotes hypertension and albuminuria during the autoimmune disease systemic lupus erythematosus. Hypertension, 59, 673-679.

O’Donovan, A., Tomiyama, A. J., Lin, J., Puterman, E., Adler, N. E., Kemeny, M., ... Epel, E. S. (2012). Stress appraisals and cellular aging: A key role for anticipatory threat in the relationship between psychological stress and telomere length. Brain, Behavior, and Immunity, 26, 573-579.

Oyama, J., Maeda, T., Sasaki, M., Higuchi, Y., Node, K., \& Makino, N. (2012). Repetitive hyperthermia attenuates progression of left ventricular hypertrophy and increases telomerase activity in hypertensive rats. American Journal of Physiology - Heart and Circulatory Physiology, 302, $\mathrm{H} 2092-\mathrm{H} 2101$.

Perez-Rivero, G., Ruiz-Torres, M. P., Rivas-Elena, J. V., Jerkic, M., Diez-Marques, M. L., Lopez-Novoa, J. M., ... Rodriguez-Puyol, D. (2006). Mice deficient in telomerase activity develop hypertension because of an excess of endothelin production. Circulation, 114, 309-317.

Puig, O., Wang, I. M., Cheng, P., Zhou, P., Roy, S., Cully, D., ... Cai, T. Q. (2010). Transcriptome profiling and network analysis of genetically hypertensive mice identifies potential pharmacological targets of hypertension. Physiological Genomics, 42A, 24-32.

Richter, T., \& von Zglinicki, T. (2007). A continuous correlation between oxidative stress and telomere shortening in fibroblasts. Experimental Gerontology, 42, 1039-1042.

Schlager, G. (1974). Selection for blood pressure levels in mice. Genetics, 76, 537-549.

Schlager, G., \& Sides, J. (1997). Characterization of hypertensive and hypotensive inbred strains of mice. Laboratory An imal Science, 47, 288-292.

Sonawane, P. J., Sahu, B. S., Sasi, B. K., Geedi, P., Lenka, G., \& Mahapatra, N. R. (2011). Functional promoter polymorphisms govern differential expression of HMG-CoA reductase gene in mouse models of essential hypertension. PLoS One, 6(1), e16661.

Taylor, N. E., Glocka, P., Liang, M., \& Cowley, A. W. Jr. (2006). NADPH oxidase in the renal medulla causes oxidative stress and contributes to salt-sensitive hypertension in Dahl S rats. Hypertension, 47, 692-698.

Valdes, A. M., Andrew, T., Gardner, J. P., Kimura, M., Oelsner, E., Cherkas, L. F., ... Spector, T. D. (2005). Obesity, cigarette smoking, and telomere length in women. Lancet, 366, 662664.

Wong, C., Mahapatra, N. R., Chitbangonsyn, S., Mahboubi, P., Mahata, M., Mahata, S. K., \& O’Connor, D. T. (2003). The angiotensin II receptor (Agtr1a): functional regulatory polymorphisms in a locus genetically linked to blood pressure variation in the mouse. Physiol Genomics, 14(1), 83-93. 
Yang, Z., Huang, X., Jiang, H., Zhang, Y., Liu, H., Qin, C., ... Ju, Z. (2009). Short telomeres and prognosis of hypertension in a Chinese population. Hypertension, 53, 639-645.

Ye, S., Ozgur, B., \& Campese, V. M. (1997). Renal afferent impulses, the posterior hypothalamus, and hypertension in rats with chronic renal failure. Kidney International, 51, 722-727.

Zhao, J., Miao, K., Wang, H., Ding, H., \& Wang, D. W. (2013). Association between telomere length and type 2 diabetes mellitus: A meta-analysis. PLoS One, 8, e79993. 